EPJ Web of Conferences 33, 01008 (2012)

DOI: $10.1051 /$ epjconf $/ 20123301008$

(C) Owned by the authors, published by EDP Sciences, 2012

\title{
Evaluation of the Impact of Wind Generation on the Electricity Market Prices and on the Profitability of New Wind Investments
}

\author{
A. J. Pereira ${ }^{1, a}$, J. T. Saraiva ${ }^{2, b}$ \\ ${ }^{1}$ Inst. Politécnico de Coimbra, Inst. Superior de Engenharia de Coimbra, Rua Pedro Nunes, 3030 - \\ 199 Coimbra, Portugal \\ ${ }^{2}$ INESC Porto, Dept. Eng. Electrotécnica e Computadores da Faculdade Engenharia da Universidade \\ do Porto, Rua Dr. Roberto Frias, 4200 - 465 Porto, Portugal
}

\begin{abstract}
This paper describes a Dynamic Model of the electricity sector that can be used to simulate the evolution of some key variables on the long term, namely the evolution of the electricity price, of the demand and of the capacity factors of the technologies in the generation mix. This model can be used in different ways and by several agents, for instance to estimate the impact on the electricity price of the increasing presence of renewable power stations, namely using wind power and PV systems. In several countries these stations are paid feed-in tariffs with a fixed price but in some cases this scheme is under discussion and there are opinions that payments determined by the market price are more adequate and would bring fewer costs to final consumers. Such a change has to be carefully evaluated given that the presence of renewable stations bidding at an infra marginal price will affect the price itself. The model described in this paper can be used in a profitable way both by governmental agencies when preparing or studying alternative remuneration schemes to renewable stations or by promoters themselves to get more insight to the profitability of their investments, namely if the fixed feed-in tariffs in force in several countries are changed.
\end{abstract}

\section{Introduction}

In recent years the consciousness that electricity should be as much as possible generated using renewable sources has been growing. This concern is driven by several factors, namely environmental aspects and the desire to turn countries more independent regarding fossil fuels. On the other hand, the development of renewable sources, as for instance wind generation, is creating new industrial and research activities and it contributes to create jobs and develop new technologies. In order to reach this objective, several countries adopted schemes to subsidize these investments, thus supporting part of the risk associated to this activity. Other countries adopted feed-in tariffs that subsidize the electricity generated using these renewable resources, either under the form of pure fixed prices set by some administrative or governmental agency, or corresponding to variable tariffs

\footnotetext{
a e-mail : ajcp@isec.pt

be-mail : jsaraiva@fe.up.pt
}

This is an Open Access article distributed under the terms of the Creative Commons Attribution License 2.0, which permits unrestricted use, distribution, and reproduction in any medium, provided the original work is properly cited. 
associated, for instance, to the market price plus a premium over it. The costs inherent to these subsidies are internalized in the final end user tariffs and these schemes proved along the last two decades to be very attractive in the sense that they have positively contributed to induce investments in wind parks and photovoltaic stations in countries as Portugal, Spain and Germany. For illustrative purposes and as a result, the installed capacity in wind parks in Portugal corresponded to about 4000 MW out of 17.000 MW in the beginning in 2011 and recent figures indicate that last November there were peaks of more than $50 \%$ of wind power penetration in the share of power to supply the national demand.

As a result of the increased participation of sources having subsidizing tariff schemes (as wind parks and PV stations, and also as cogeneration stations and small hydros), traditional generation stations (but in particular large thermal stations using coal and natural gas) are facing a reduction of their utilization factors and so their chances of being dispatched in the day ahead markets are getting reduced. Another consequence of this increased participation of subsidized sources in the generation mix is the reduction of the market clearing price. In this scope, it is becoming less uncommon to get $0.0 € \mathrm{MWh}$ clearing prices namely in valley demand periods with large amounts of wind and hydro available primary resources. This means that stations receiving the market price have a null remuneration during such hours but, on the contrary, stations subjected to pure feed-in tariffs remain immune to these difficulties while their presence contributes to the price reduction.

This scenario in which more subsidized generation contributes to reduce the market price occurs while the subsidizing schemes are under discussion in several countries, namely in Portugal, given that an increasing number of opinion makers argue that the amount of installed capacity in renewable generation is no longer compatible with the current feed in subsidized tariffs. Therefore, it is important to evaluate the impact of different possible levels of renewables in the market clearing price and to what extent this reduction can itself compromise the profitability of these units if, in the near future, the legal framework changes and, for instance, wind power is forced to bid in the market. In order to perform this evaluation, we used a long term generation planning model that was developed using System Dynamics, an approach well suited to model the long term evolution of power systems, including the demand, the average market price and interactions between several variables. This long term model will be used admitting different penetrations levels of wind power so that we can estimate the evolution of the market price. The output average prices can then be used in a more sounded way by policy makers to design new rules both to frame the participation of renewables in the generation mix and by promoters to evaluate the profitability of new investments in renewables as well as of existing traditional thermal stations.

Apart from this Introduction, Section 2 describes the long term model of the electricity sector including a brief description of System Dynamics. Section 3 details the developed generation expansion model that is used in a combined way with the long term model of Section 2. Then, Section 4 describes the solution algorithm and Section 5 illustrates the application of this approach using a Case Study that represents a scaled model of the Portuguese power system. Finally, Section 6 presents the conclusions of this work.

\section{Dynamic Model of the Electricity Sector}

\subsection{Brief description of System Dynamics}

Most economic equilibrium models assume rationality of decision-makers. This is often far from the reality because it would mean that decision-makers have complete knowledge of the problem and of meaningful information, they are able and have the time to anticipate the consequences of their decisions [1,2]. Given these characteristics, System Dynamics approaches can be summarized in the following iterative steps. In the first place, System Dynamics promotes an in-depth understanding of the problem and of the relevant relationships between variables and parameters. Then, the analyst should set the boundaries of the problem identifying the factors to include and the ones to exclude. 


\section{$2^{\text {nd }}$ European Energy Conference}

The next step corresponds to establish a reference mode for the problem as well as the time horizon of to analyse. Then, a dynamic hypothesis is developed in terms of a causal loop diagram and of stock and flow diagrams. Afterwards, the model is implemented for simulation and is tested in order to define the variables to be modeled in an endogenous way and which of them can be considered as exogenous or can be omitted. This will lead to the final definition of the boundaries of the problem so that it can adequately replicate the system under analysis. In this step it can also be conducted a sensitivity analysis to eventually help deciding if further efforts should be dedicated to increase the precision of input data. Finally, when a reasonable confidence level in the model is achieved, one can then perform simulations and studies using it.

System Dynamics models typically includes several kinds of relations and equations [2] namely state equation, representing accumulations involving the input and output variables and rate equations, that are used to control the input or output variable in a state variable equation. They also include auxiliary equations, corresponding to additional algebraic equations relating in a complex way state variables, rate variables and constants and finally table functions, representing a set of data along a specified time interval.

System Dynamics models typically display a number of characteristics such as direct description, natural and clear format, qualitative and quantitative form and robust ability to use data. As a result of these characteristics, they are used to investigate the structure, function relationship and dynamic behaviors of complex systems such as the ones mentioned in [3]. In this paper, System Dynamics is used in power markets, and the market dynamics is analysed based on the relationship among wholesale power market, power demand, power supply and the construction of new power stations.

\subsection{General structure of the dynamic model}

In recent years, System Dynamics started to be used to model the long-term behavior of electricity markets. System Dynamics was conceived by J. W. Forrester in the 1960's and [4] summarizes its main concepts. On the other hand, regarding the application of System Dynamics to power systems, references [5, 6, 7] illustrate the application of these concepts to the Generation Expansion Planning problem, GEP, that aims at identifying the most adequate set of generators, including capacities and technologies, to be added to an existing generation system in order to supply the expected demand while complying to some reliability or adequacy indices.

The GEP problem corresponds to a long run estimation determined by a number of variables that are in fact interdependent. For instance, the profit of the generation agents depends on the global economic environment, in the sense that this affects the industrial activity, the demand level and ultimately the capacity factor of each technology, in terms of the number of hours each station is operating. On the other hand, the demand depends on the price of coal and fuels and these prices also have an important impact on the electricity prices, that on its turn also influences to some degree the demand itself. Finally, investment decisions taken by other competitors as well as the evolution of the installed capacity in renewable stations, namely wind parks and hydro stations, affect the generation mix. Given the characteristics of this long term evaluation, we developed an approach fully reported in $[8,9]$ that uses System Dynamics as a way to incorporate all the dependencies and loops and to include delays between the instant in which a project is approved and it starts operation. These dependencies and interactions are illustrated in the casual diagram displayed in Figure 1.

According to this diagram, there is a positive cause-effect relation between the installed capacity and the amount of reserves. On the other hand, decommissioning units has a negative effect on the total available power and a demand increase reduces the reserve margin and eventually induces an increase in the electricity price. This diagram also includes a delay, $\tau$, between the decision to build a new unit and its commissioning. This delay is due to the licensing and the building period and it should not be forgotten when modelling the long-term evolution of the electricity price. Finally, there is a feed-back loop between the demand and the electricity price. Departing from an initial set of investment decisions, it is obtained the evolution of the electricity price using an initial demand 
rate. However, the price level will also impact on the demand depending on its elasticity. If there is a capacity shortage or a very dry year, the electricity price tends to increase which can induce a demand reduction. The evolution of fuel costs and the updated electricity prices can then originate a change in the investment plans because the profitability of the expansion projects will change.

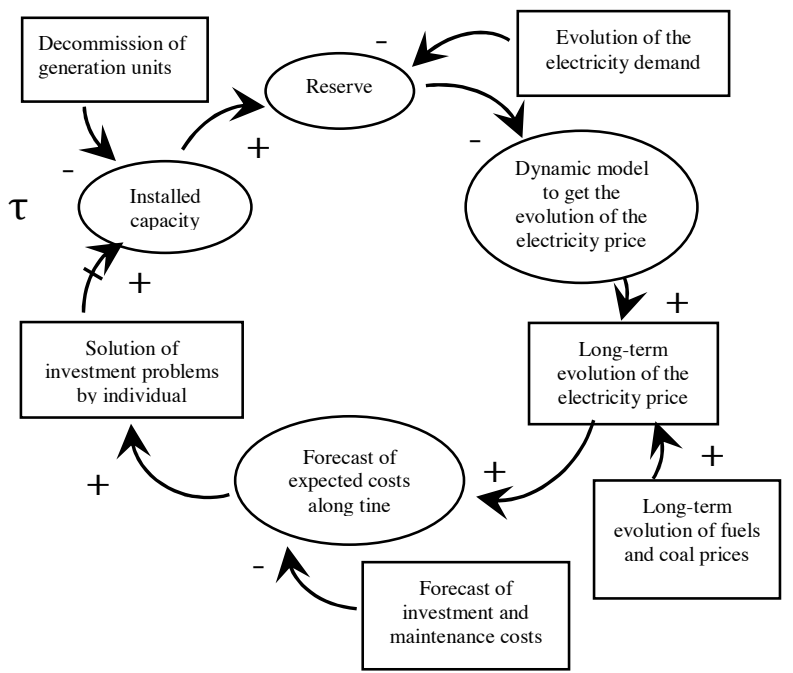

Fig. 1. Casual diagram representing the electricity market long-term interactions.

The dynamics of an electricity market is described by a set of differential equations that consider system feedbacks, delays, stock-and-flow structures and non-linearities. The evolution of the market is determined by modelling the variables that have a direct influence on the changes that can affect supply and demand. The developed model corresponds to a system dynamic representation of an electricity market focusing on the supply of different competing generation technologies. For illustration purposes, we can consider technologies as wind power, hydro stations and thermal stations including, for instance, both coal and natural gas. These technologies have very different economical, technological and environmental characteristics as well as investment and operational costs, operational characteristics, typical emission levels and potential for technological progress.

Modeling the dynamics of electricity markets and generation systems can be organized in three main blocks as follows:

- taking into account the demand and existent power plants, the first block estimates the electricity prices, the power outputs and the capacity factor for every technology along the planning horizon;

- the second block aims at obtaining an expansion plan of the generation system, namely including the type of stations, the commissioning dates and their installed capacities. This expansion plan is prepared using the electricity prices and the capacity factors estimated in the previous block and it can either correspond to a plan built by a governmental agency for reference purposes or result from the planning actions of individual generation companies. Section 3 details the generation planning model that was developed in the scope of this research;

- finally, the available capacity is determined by the additions of new capacity considering a specified delay and the decommissioning of old power plants. The generation mix along each year of the planning horizon together with the estimated demand can now be used to estimate the evolution of some adequacy indices to characterize the risk of the system not being able to meet the demand. If below than a specified threshold, we iterate again going back to the first block in order to update the estimates of the electricity price and of the capacity factors considering the results of the expansion planning exercise. 
When running this simulation, it is important to consider a long term planning horizon in order to give long-term impacts time to produce their influence. On the other hand, the time resolution should be sufficiently small in order to enable capturing the short-term mechanisms included in the model. In the simulations described in Section 5 we adopted a 15 -year time horizon, in order to allow the resource availability and technological progresses to have impact and 1 hour for the time resolution so that electricity prices can adjust the demand/supply balance over the year. The next subsections detail the dynamic model of the electricity sector that outputs the estimates of the electricity price, of the demand and of the capacity factors of each technology in the generation mix. These estimates are then used to prepare the expansion plans of each generation agent described in Section 4.

\subsection{Simulation of the generation system}

The generation system is simulated considering the existing stations in the initial year and the ones to be commissioned and decommissioned along the horizon. In order to simulate the electricity generation, the model considers thermal, hydro and wind power stations. Regarding wind parks, we considered the total installed capacity and the average annual number of hours that these stations generate. In this scope, we used typical values of 20 to $25 \%$ for their capacity factors, measuring the generated energy regarding what could be generated if the wind parks were at full capacity all along the year. As wind generation fluctuates in an intermittent way, we used a stochastic process to distribute the generation of wind parks along each year. This certainly increases the realism of the model and of the influence of this generation in electricity prices. For the wind parks we did not specify Forced Outage Rate, FOR, values because we admitted that the mentioned capacity factors of $20 \%$ to $25 \%$ already reflect both the availability of the primary resource and of the stations themselves.

Hydro stations were grouped in two sets: run-of-river and reservoirs. Using historical values, we set the capacity factor of run-of-river stations at $25 \%$. However, this value can still vary from dry to wet years and so we considered a stochastic process with a mean value of $25 \%$ and a standard deviation of $5 \%$. For reservoirs, we adopted a similar approach but using a mean value of $20 \%$. Similarly to wind parks, the FOR of hydro stations was not specified because the capacity factors already internalize the availability of the stations.

Finally, thermal stations were grouped in different sets according to their technologies. This means the model can group thermal stations in coal and CCGT, for instance, and eventually consider subgroups in each of them if that is required to model adequately the operation costs. The generation of these stations will depend on the amount of electricity coming from the wind parks and hydro stations and on the evolution of the demand along the horizon. For each technology, the demand to be supplied by thermal stations is then allocated considering the corresponding operation costs. For each technology, the cost curve was organized in steps for ranges of the capacity factor as it is illustrated in Figure 2. This information from each technology, together with the corresponding installed capacity and the demand to be supplied by thermal stations is used to make the dispatch of these stations.

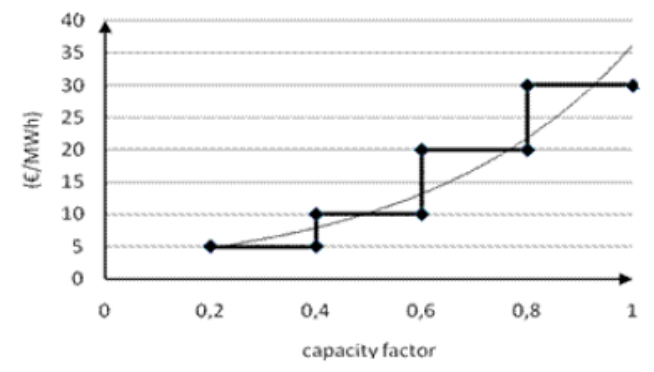

Fig. 2. Illustration of a cost curve of a thermal station. 
Regarding the investments to be selected in the scope of the long term simulation, we admitted that wind generation is paid feed-in tariffs as it currently happens in a large number of European countries and that, in any case, it has priority over other more classical technologies. On the other hand, we also admitted that new wind power capacity is allocated in the scope of tenders conducted by state agencies, so that these capacity expansion investments follow a different reasoning when compared to investments in classical thermal technologies as CCGT or coal stations. Similarly, for hydro stations it is also common to allocate new capacity in the scope of public tenders. Given that the operation cost of these stations is very reduced, these investments are typically very attractive. Some preliminary experiments admitting hydro stations as a candidate technology in the long term dynamic simulation showed that generation agents would decide to invest as much as possible in hydro stations as early as possible in the planning horizon. To overcome this effect, we admitted that there was a commissioning schedule for hydro stations coming from the mentioned public tenders that was used to determine the years in which new hydro capacity was added to the generation mix. In this sense, hydro stations were not admitted as candidate technologies, but in fact the hydro system evolves as specified according to the mentioned scheduled.

\subsection{Simulation of the demand evolution}

The evolution of the demand along the planning horizon is determined by the evolution of the demand rate and of the electricity price, namely because the model includes information about the elasticity of the demand to the price. To start with, the demand rate is modeled by an OrneistenUhlenbeck Mean Reverting Process as detailed in [7]. According to this model, the demand rate incorporates a long term trend and a short term component. This short term component determines variations that superimpose on the long term trend but, as the time evolves, it tends to attenuate and the demand rate reverts again to the long term trend. These two terms are modeled by $F_{R}$ and by $d z$ in (1). The first term is given by (2) and it is influenced by the mean value to which the demand rate reverts on the long term, drate $_{L T}$, and by the speed of reversion, $\eta$. This parameter is specified by the user and it models the velocity regarding which variations dissipate and the demand rate returns back to its long term mean value. The second term is given by (3) using a Wiener process that depends on the volatility parameter $\delta$. If $\delta$ increases, the demand tends to display larger variations although having the same mean value. In the scope of the dynamic model, we start at a reference demand rate drate $_{0}$ and then for each step in the simulation we estimate the evolution of the demand rate using (4). Finally, in these expressions $\varepsilon_{\mathrm{t}}$ represents a random number extracted at each time step from a normal distribution $(0,1)$.

$$
\begin{gathered}
d x=F_{R}+d z \\
F_{R}=\eta \cdot\left(\text { drate }_{L T}-\text { drate }_{t}\right) \cdot \Delta_{t} \\
d z=\varepsilon_{t} \cdot \delta \cdot \sqrt{\Delta t} \\
\text { drate }^{=} \text {drate }_{0}+\int_{0}^{T} d x \cdot d t
\end{gathered}
$$

For each time in the horizon, the estimated demand rate is then used to obtain the demand using (5). This expression indicates that the demand in period t depends on the demand rate and on the reference demand set for the starting year. Finally, as indicated above, the demand shows some elasticity to the electricity price, although not very large. This effect is modeled by (6) in which $\pi^{\mathrm{t}}$, $\pi^{0}$ and $E_{\mathrm{d} \pi}$ represent the electricity price in period $t$, the electricity price in the starting period and the elasticity of the demand to the price. 


$$
\begin{gathered}
d_{r e f, t}=d_{r e f 0}+\int_{0}^{T} \text { drate }_{t} d_{r e f 0} \cdot d t \\
d_{\text {elec }, t}=d_{r e f, t} \cdot\left(\frac{\pi^{t}}{\pi^{0}}\right)^{E_{d \pi}}
\end{gathered}
$$

\subsection{Simulation of the electricity price evolution}

The evolution of the electricity price along the horizon is determined by the price set for the starting period of the simulation, $\pi^{0}$, and also by the relation between the demand and the installed capacity in each period. Using (7), the electricity price variations depend on the difference between the demand in period $t$ and the installed capacity in the sense that if there is a lack of investments determining an excess of demand regarding the installed capacity, the difference in (7) is positive originating a positive variation of the electricity price using (8). In any case, we admit there is a delay before such differences eventually determines a reaction of the market. These differences between the demand and the installed capacity are smoothed by the Attenuation Factor, AF, used in (7). Conversely, if the installed capacity shows an excess regarding the demand, this contributes to induce a reduction of the electricity price.

$$
\begin{gathered}
\Delta \pi^{t}=\pi^{0}\left(\frac{d_{\text {elec }, t}-G_{\text {total }}}{d_{\text {elec }, t}}\right) \cdot \frac{1}{A F} \\
\pi^{t}=\pi^{0}+\int_{0}^{T} \Delta \pi^{t} \cdot d t
\end{gathered}
$$

\subsection{Global dynamic model of the electricity sector}

The solution of the investment problems to be detailed in Section 3 requires the knowledge of the evolution of the electricity price and of the capacity factors of each technology. These inputs are provided by the long term model of the electricity market developed using System Dynamics. Figure 3 presents an aggregated view of this model.

The generation system is modeled in the lower part of this model considering nodes that aggregate the generation coming from the technologies in the mix. In this case, we considered nodes to model the injections from wind parks (lower left side of Figure 3), hydro stations decomposed in run of river and in reservoirs (lower central part of Figure 3) and thermal stations also grouped according the technologies in the mix and eventually further subdivided according to their generation costs (lower right side of Figure 3). Then, the generation obtained in each time step from each of the components of the mix is added in the node "Total Production" and this value is multiplied by a factor larger than 1.0 (typically in the range from 1.07 to 1.1 in well developed systems) to account for active losses in transmission and distribution networks.

The demand model detailed in Section 2.4 provides for each time step and estimate of the electricity demand and this estimate together with the generation determines the evolution of the electricity market price according to the sub model in Section 2.5. As a result of these interactions, the dynamic model also provides the dispatch of the thermal technologies using the specified operation costs and the estimated capacity factors.

When this long term simulation ends, we get final estimates for every period in the horizon of the electricity demand, of the electricity market price and of the capacity factors of the different technologies in the generation mix. These estimates are then used by each generation agent to run their capacity expansion problems as it will be detailed in Section 3 . 


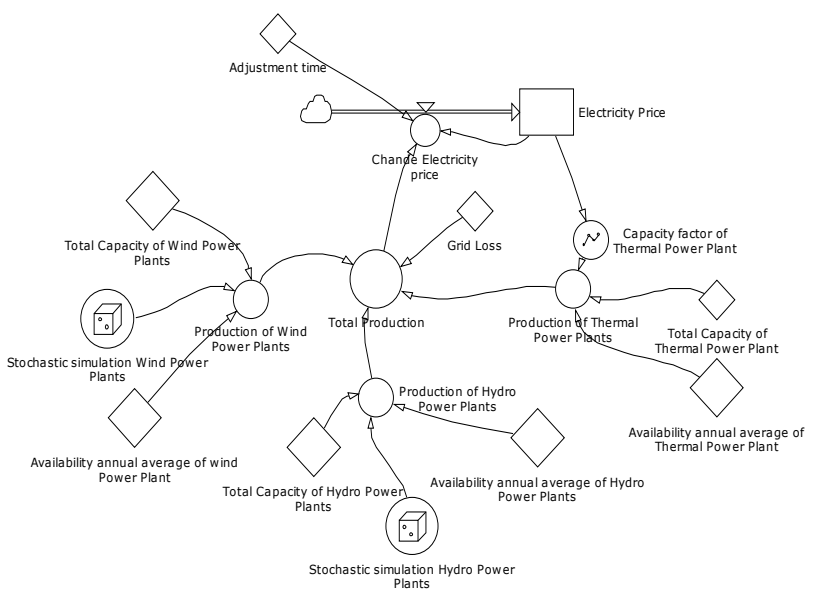

Fig. 3. Dynamic model to simulate the electricity price.

This figure also shows that the dynamic model of the electricity market includes a feed-back loop involving the electricity price, the output of each generation technology and the demand. In order to clarify how these interrelations are addressed, let us consider that the dynamic simulation is initialized with a set of values for the electricity price (for instance, available from an historic data set), as well as sets of initial values for the demand and for the capacity factors of the hydro and wind generation (also based on historic values). Using these initial values, the individual profit maximization problems of each generation company (to be detailed in Section 3) are solved. As a result, we get schedules for the additions of new thermal capacity so that the installed capacity along the horizon is updated. Then, from the historic data we estimate the generation from the hydros and special regime sources so that we obtain the demand to be supplied by the thermal stations. Using the set of initial electricity prices and the cost curves of each thermal technology we estimate the corresponding capacity factors. The generation from thermal stations in each time interval is added to the outputs of the hydros and the special regime sources, it is multiplied by a factor larger than 1.0 to consider transmission and distribution losses and finally the global generation and the demand in each time interval are used in the Stocks and Flows diagram in the top of Figure 3 to compute a price mismatch that is mathematically modelled by (7) and that leads to the new set of prices along the horizon given by (8). If there is a positive mismatch between the demand and the total installed capacity, then the price should be increased leading to a progressive increase of the capacity factors of the thermal stations. At the long run, as the simulation evolves, we will tend to an equilibrium between the demand and the generation.

The implemented dynamic model also includes a loop to represent the interrelation between the demand and the electricity price. According to Section 2.4, we specify a long term demand rate but then along the simulation the demand evolution is adjusted considering this relation with the price. This loop is not displayed in Figure 3 and a more detailed description of this dependency can be obtained in [9]. Mathematically this loop is modelled by (4), (5) and (6). In (5) we use a demand value specified in the beginning of the simulation. The demand rate is estimated using (4) and using this information we update the reference demand in each step of the horizon using (5). Finally, this reference demand is used to obtain the demand in each step considering the elasticity of the demand to the price, modelled by (6). 


\section{Generation Expansion Planning Model}

The investment decision process in power generation changed with the introduction of competition in the electricity generation sector because investments on new generation capacity became a commercial and risky activity. This is because investors are more interested in short-term investment return and are reluctant to invest on generation capacity that requires large investment while implying long pay-back periods. On the other hand, this process has increasing uncertainties coming from the load behaviour, restructuring policy and market management rules which can influence the revenues. Investors are expected to spend a considerable amount of time and effort in analyzing the interaction between investment and the decentralized decisions by participants. In taking a generation investment decision, expectations concerning future electricity demand, market prices, changes of regulatory policies, as well as the financial environment are major considerations.

In the developed formulation of the GEP in a restructured electricity market, the objective is to maximize the total expected profit of each individual generation company over a planning horizon (9), while guaranteeing the safe operation of the power systems through the competition between generation companies [10,11]. The developed formulation incorporates the volatility of market prices of electricity and fuels and load growth. The expected revenues are based on the predicted market price, construction costs, fixed O\&M costs, typical capacity factors for each technology and expected operation cost. Due to the volatile nature of the market, some sources of uncertainty in future operating conditions such as the forecasted market price of electricity, load growth rates, fuel costs and equipment availability are also considered in the planning exercise. The GEP problem stated for a generation company $i$ in a competitive environment can be formulated according to (915). In this problem the decision variables are $X_{t}^{i, j}$ and they represent the new installed capacity of the generation agent $i$ of technology $j$ in period $t$.

$$
\begin{aligned}
\max z= & \sum_{t=1}^{T}\left[\pi^{t} \sum_{j=1}^{M} C C_{t}^{i j} \alpha_{t}^{i j}-\sum_{j=1}^{M}\left(\operatorname{Cinv}_{t}^{j} \cdot X_{t}^{i j}+\operatorname{Cop}_{t}^{j} \cdot \operatorname{CC}_{t}^{i j} \cdot \alpha_{t}^{i j}\right)\right] \\
\text { subj } \quad & X_{t}^{i j} \leq M I C_{t}^{i j} \\
& \sum_{j=1}^{M} X_{t}^{i j} \leq M I C_{t}^{i} \\
& \sum_{j=1}^{M} X_{t}^{i j} \cdot \operatorname{Cinv}_{t}^{j} \leq M X I N V_{t}^{i} \\
& \sum_{t=1}^{T} \sum_{j=1}^{M} X_{t}^{i j} \cdot \operatorname{Cinv}_{t}^{j} \leq M X I N V^{i} \\
& C C_{t}^{i j}=C C_{t-1}^{i j}+X_{t}^{i j} \\
& t=1, \ldots ., T ; \quad i=1, \ldots ., N ; \quad j=1, \ldots ., M
\end{aligned}
$$

In this formulation:

$T \quad$ number of stages in the planning horizon;

$t \quad$ stage in the planning horizon (year);

$N \quad$ number of GENCOs;

$i \quad$ investment index for GENCO $i$;

$M \quad$ number of candidate technologies;

$j \quad$ type of candidate expansion technology;

$\pi^{t} \quad$ price of electricity in stage $t$;

$\alpha_{t}^{i j} \quad$ capacity factor in stage $t$ for GENCO $i$ and technology $j$;

Cinv $_{t}^{j} \quad$ investment cost for technology $j$ at stage $t$; 


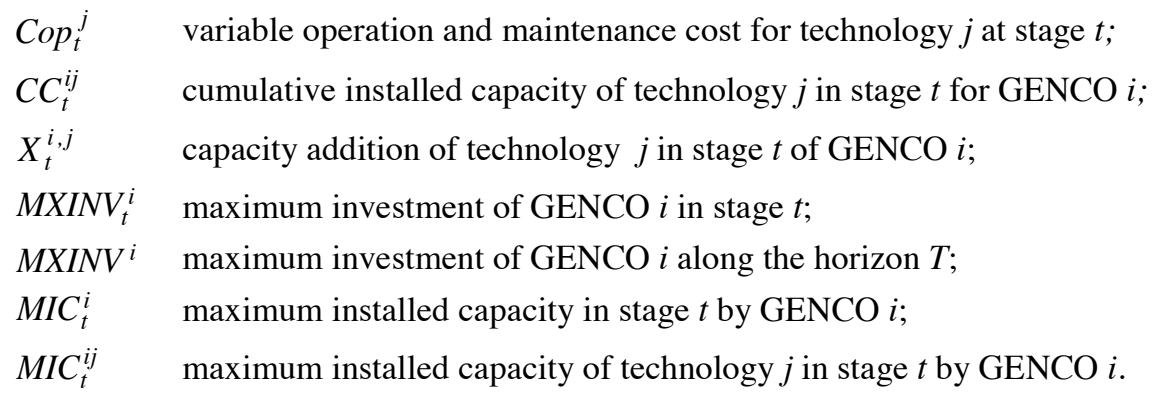

In this formulation, the objective function maximizes the profit of each generation company $i$ and it includes three terms as follows. The first term represents the revenues of the generation agent $i$ from selling electricity in each period $t$ at the electricity market price $\pi^{t}$. Apart from this price, this term includes the installed capacity of technology $j$ in period $t$ and the capacity factor of this technology in this period, in terms of the number of hours it was dispatched in the market. The second term models the investment cost due to the investment in period $t$ in power stations of technology $j$ and with capacity $X_{t}^{i, j}$. Finally, the third term represents the operation costs associated with each technology $j$, and the value of these costs depends on the capacity factor of each technology, which in turn depends on the demand level and on the generation mix.

This objective function is subjected to a number of constraints as follows. Constraints (10) limit the new installed capacity of technology $j$ that each generation agent can add to its mix in each period $t$. Constrains (11) impose an upper bound to the total capacity in all candidate technologies that each generation agent can include in the mix in period $t$ and constraints (12) limit the investment cost in each period $t$ to an upper bound. Constraints (13) imposes an upper bound to the global investment of this generation agent over the entire planning horizon and (14) simply accumulates the installed capacities of each generation agent in terms of each candidate technology as the planning horizon develops.

This problem has a discrete combinatorial nature given that each agent has a limited number of candidate technologies and for each of them there will typically be a number of available normalized capacity values that can be selected. This means that the decision variables $X_{t}^{i, j}$ can typically take values from a pre specified list that should be prepared by the planner in the sense that it contains the admissible capacities for each technology, and the estimates of the corresponding investment and operation costs. This problem can then be solved using Genetic Algorithms as described in [10]. In this formulation, a generation company first decides its new capacity investments based on its own decision criteria and the decisions of individual generation companies are then aggregated in order to assess the adequacy of the future generation system and also in view of global objectives of energy policy set at a state level as it will be detailed in the next Section.

\section{Solution Algorithm}

The solution of the individual generation expansion problems detailed in Section 3 requires some inputs namely the expected evolution of the electricity price and of the capacity factor of each technology in the generation mix along the planning horizon. These values can be provided by the long term dynamic model of the electricity sector as described in Section 2. As a result, we implemented a solution algorithm in which the two problems are solved in sequence until a convergence criterium becomes valid. The next paragraphs detail the main steps of this iterative process:

i) in the first place, we set initial values for the electricity price, for the demand and for the capacity factor of each technology for each year in the planning horizon; 
ii) secondly, each generation company solves its own profit maximization problem taking into account its current generation mix and its available financial resources. This corresponds to solve as many problems (9-15) as the generation agents and the results correspond to the additions that each agent considers as more adequate, including the selected technologies, the installed capacities and the commissioning years;

iii) thirdly, the partial plans obtained in step ii) are aggregated in order to obtain the global evolution of the generation mix. Using this generation mix and the estimated demand evolution, it is possible to evaluate a number of global constraints regarding, for instance, the adequacy of the generation system and the compliance with general targets established at the national energy policy namely regarding minimum or maximum values set for the share of some particular technologies. In particular, adequacy can be evaluated using the reserve margin (in terms of the percentage of excess of the installed capacity regarding the peak power) and a reliability index as the Loss of Load Expectancy, LOLE, for which Grid Codes in several countries typically set a maximum number of hours during which the demand may not be attended. The reserve margin and the LOLE are computed for each year in the planning horizon and are compared with the specified limits;

iv) if at least one of these constraints does not hold, the dynamic simulation of the electricity system is activated again using the additions identified in the expansion plans of the individual companies. This will update the electricity price, the demand and the capacity factor of each technology along the planning horizon so that this information is returned back to the generation companies for them to solve again their individual profit maximization problems, that is, the algorithm returns to step ii);

v) this iterative process will end when a convergence criterium is meet, namely when all the global constraints are meet.

This algorithm was implemented in MATLAB, POWERSIM and Microsoft Excel, as follows. The individual profit maximization expansion problems were implemented in MATLAB using Genetic Algorithms as detailed in $[9,10]$. MATLAB was also used to compute the value of LOLE in each year of the planning horizon considering the demand evolution and the generation mix resulting from the generation system existing in the starting year plus the additions obtained by the individual expansion problems. Finally, the long term dynamic simulation model was implemented and solved using the software package POWERSIM [12, 13]. In this application the differential equations included in the model were solved using a numerical technique considering a 1 hour integration step. This package then allows aggregating the hourly outputs, for instance in average weekly or monthly values in order to turn the comparisons easier. Finally, Microsoft Excel was used as an interface between these two modules, to specify data and to receive results, prepare graphs and output tables.

\section{Case Study}

\subsection{Data}

The long term simulation model of the electricity sector will now be illustrated using a Case Study based on the Portuguese generation system. In this study we used data publicly available in the web site of the Portuguese TSO (www.ren.pt) together with historic series of hourly electricity prices from the Common Iberian Electricity Market Operator (www.omie.es). The departing system was defined scaling the Portuguese system to one half given that the demand reached $50.000 \mathrm{GWh}$ in 2009 and we admitted a demand in the departing year of the planning horizon of $25.150 \mathrm{GWh}$. Finally, and before detailing the date used in this study we admitted that there were three competing generation companies, five existing technologies and three candidate technologies.

The generation system in the departing year has an installed capacity of $6.200 \mathrm{MW}$ among which $4850 \mathrm{MW}$ are thermal as indicated in Table 1. Apart from the number of units of each technology, 
this table also indicates the installed capacity, the operation cost and the FOR of each unit, as this reliability index is required to calculate the LOLE index. For illustration purposes, it is also important to notice that we admitted that coal stations were grouped in two sets so that we can specify different operation costs for each group. The generation mix also includes wind parks and hydro stations, grouped in run-of-river and reservoirs. In the departing year, the installed capacity in run-of-river stations was $300 \mathrm{MW}$, in reservoirs was $450 \mathrm{MW}$ and in wind parks was $600 \mathrm{MW}$. Finally, regarding the existing generation mix, Table 2 details the portfolio of each of the three generation companies. In the departing year, GENCO 1 owns $24,2 \%$ of the installed capacity, GENCS 2 owns $21,8 \%$ and GENCO 3 owns $23,4 \%$. For illustration purposes we admitted that the remaining 30,6\% were owned by other agents not interested in expanding their portfolios. These can correspond for instance to industrial companies owning cogeneration stations as well as small companies owning small hydro systems or wind parks.

Table 1. Characteristics of the existing five thermal technologies.

\begin{tabular}{|c|c|c|c|c|}
\hline $\begin{array}{c}\text { no. } \\
\text { units }\end{array}$ & Technology & $\begin{array}{c}\text { Generating } \\
\text { Size (MW) }\end{array}$ & $\begin{array}{c}\text { Operation Cost } \\
(€ / M W . h)\end{array}$ & FOR \\
\hline 3 & Coal_1 & 300 & 25.25 & 0.02 \\
\hline 2 & Coal_2 & 400 & 28.00 & 0.02 \\
\hline 5 & Gas turbine & 250 & 35.75 & 0.01 \\
\hline 2 & Oil turbine & 200 & 40.00 & 0.03 \\
\hline 6 & CCGT & 250 & 26,75 & 0.01 \\
\hline
\end{tabular}

Table 2. Portfolios of the three competing generation agents.

\begin{tabular}{|c|c|c|c|}
\hline Technology & GENCO_1 & GENCO_2 & GENCO_3 \\
\hline Coal_1 (MW) & 300 & 300 & - \\
\hline Coal_2 (MW) & 400 & - & 400 \\
\hline Gas turbine (MW) & 250 & 250 & 500 \\
\hline Oil turbine (MW) & 200 & 200 & - \\
\hline CCGT (MW) & 250 & 500 & 250 \\
\hline Wind parks (MW) & 100 & 100 & 200 \\
\hline Hydro (MW) & 0 & 0 & 100 \\
\hline
\end{tabular}

The input data also includes information about the load diagram, the candidate technologies, parameters set for the dynamic model and additional information about the financial resources of each agent, about the decommissioning plan and about the scheduled investments in hydro stations and wind parks, given that these investments are to some extent subjected to a different decision process. To start with, the demand in the starting period was modelled considering a load duration curve with a peak power of $3900 \mathrm{MW}$ and having 6 steps. For each of them, it is specified the respective power as a percentage of the mentioned peak power and the percentage of the hours of the year during which the power is at least larger than the one indicated for this time step. In this case, we adopted the following steps:

- $100 \%$ of the peak power (3900 MW) during $5 \%$ of the year;

- $90 \%$ of the peak power (3510 MW) during $20 \%$ of the year;

- $80 \%$ of the peak power (3120 MW) during $45 \%$ of the year;

- $70 \%$ of the peak power $(2730 \mathrm{MW})$ during $65 \%$ of the year;

- $60 \%$ of the peak power (2340 MW) during $85 \%$ of the year;

- $50 \%$ of the peak power (1950 MW) during $100 \%$ of the year, that is for 8760 hours.

Along the entire planning horizon the shape of the load duration curve was assumed constant. For each year of the horizon, the long term dynamic simulation will provide the evolution of the peak demand which means that using the above indications we can then adjust the power values associated to this curve while maintaining the mentioned percentages. 
Regarding the three candidate technologies, Table 3 characterizes them in terms of the available capacities, of the investment and operation costs and of the Forced Outage Rate, FOR, of the groups of each technology. It was also admitted that the construction period was 2 years regardless of the technology, supposing that all the licensing process was already completed, that is, admitting there were a number of possible sites regarding which all licenses were previously obtained.

Table 3. Characterization of the three candidate technologies.

\begin{tabular}{|c|c|c|c|c|}
\hline $\begin{array}{c}\text { Type of } \\
\text { technology }\end{array}$ & $\begin{array}{c}\text { Available } \\
\text { capacities } \\
\text { (MW) }\end{array}$ & $\begin{array}{c}\text { Investment } \\
\text { cost }(€ / \mathrm{MW})\end{array}$ & $\begin{array}{c}\text { Operation } \\
\text { Cost } \\
(€ / \mathrm{MW} . \mathrm{h})\end{array}$ & FOR \\
\hline Tech_1 & $\begin{array}{c}100 \text { or } 150 \\
\text { or } 200\end{array}$ & 500000 & 32.00 & 0.01 \\
\hline Tech_2 & $\begin{array}{c}100 \text { or } 125 \\
\text { or } 150\end{array}$ & 800000 & 28.09 & 0.02 \\
\hline Tech_3 & $\begin{array}{c}100 \text { or } 150 \\
\text { or } 200\end{array}$ & 1000000 & 24.10 & 0.02 \\
\hline
\end{tabular}

Regarding the specific data required by the dynamic model, we set the demand rate in the initial period of the horizon $t_{0}$ at $3 \%$, the long term demand rate $t_{L T}$ at $4 \%$, the electricity price in the departing year at 40,0€/MWh, the elasticity of the demand to the electricity price at 0,25 , the mean reversion parameter and the volatility of the Ornstein-Uhlenbeck process modelling the demand rate evolution at 0,40 and at 0,50. Finally, the planning horizon includes 15 years and along them we admitted there is a decommissioning plan of existing power stations as follows. In the 5th year of the horizon, $300 \mathrm{MW}$ of coal type_1 and $250 \mathrm{MW}$ of CCGT will be decommissioned. In the $10^{\text {th }}$ year all the capacity using oil (400 MW) will be decommissioned together with $200 \mathrm{MW}$ of coal type_2 and $250 \mathrm{MW}$ of gas turbines and in the 15th year, $300 \mathrm{MW}$ of coal type_1, $200 \mathrm{MW}$ of hydro reservoirs and $200 \mathrm{MW}$ of installed capacity in wind parks will be decommissioned.

On the other hand, as mentioned in Section 2.3 we admitted that investment decisions on hydro generation follow a different rationale namely due to the limited number of available places to locate new hydro stations and given their reduced operation cost. As a result we considered a schedule regarding new hydro stations established out of the generation expansion planning problem, that is, we admitted that investments in new hydro stations would always be so interesting to generation companies that the available capacity would always be entirely allocated for instance in the scope of public tenders organized by state agencies. In this case study, we admitted that $200 \mathrm{MW}$ of new hydro reservoirs will be commissioned in the $4^{\text {th }}$ year and $100 \mathrm{MW}$ of run-of-river in the $8^{\text {th }}$ year. A similar reasoning applies to new wind parks, in this case due to the subsidized tariffs in force for them. Accordingly, we considered that $100 \mathrm{MW}$ will be installed in the $2^{\text {nd }}$ year, $100 \mathrm{MW}$ in the $4^{\text {th }}$ year, $150 \mathrm{MW}$ in the $7^{\text {th }}$ year, $100 \mathrm{MW}$ in the $10^{\text {th }}$ year and $150 \mathrm{MW}$ in the $12^{\text {th }}$ year.

In order to turn the models more realist, we considered a number of additional features for instance related with the willingness of investing or not investing in a particular technology and also related with the specification of financial limitations of each GENCO. These limitations are then modelled by constraints $(12,13)$ in the generation expansion model, written for each period in the horizon, for sets of periods or eventually for the entire planning horizon, depending on the available information. In this case, we used the following information:

- GENCO 1 has no particular preference for any candidate technology and in the first set of 5 years in the horizon has $500 \mathrm{M} €$ available and more $500 \mathrm{M} €$ in the remaining 10 years;

- GENCO 2 is only interested in investing in technologies 1 and 2 and has $350 \mathrm{M€}$ available in the first 5 years, $350 \mathrm{M} €$ from the $6^{\text {th }}$ to the $10^{\text {th }}$ year and $350 \mathrm{M} €$ in the last 5 years of the horizon;

- GENCO 3 showed no preference for any particular technology and has $400 \mathrm{M€}$ available during the first 5 years, $400 \mathrm{M} €$ more from the $6^{\text {th }}$ to the $10^{\text {th }}$ year and $400 \mathrm{M} €$ more in the last 5 years; 
Finally, as a way to limit market power we specified that no generation agent can own more than $40 \%$ of the total installed capacity. Regarding the reliability and adequacy limits, we specified that the reserve margin between the total installed capacity and the peak power in each year of the horizon should range from 20 to $40 \%$ and the maximum value of the LOLE index was set at $2 \mathrm{~h}$ /year.

\subsection{Results - Reference Case}

In the first place, we ran the long term simulation of the electricity market using the data detailed above and the algorithm described in Section 4. In each step of this long term simulation, we ran the profit maximization problems as explained in Section 3 in order to build generation expansion planning problems. This first exercise will provide reference results that can then be used for comparison purposes when conducting sensitivity studies, namely considering the increase of the installed capacity in wind parks.

Regarding the results that were obtained, Figures 4 and 5 display the expansion plans built GENCO's 1 and 2 indicating the new capacity additions for each of the three candidate technologies along the planning horizon. It is important to notice that in both cases there is a concentration of investments in years 1 and 2, in years 5 and 6 and in years 11 and 12. These years coincide with the beginning of sets of five years in which each we admitted that generation companies have more financial resources to invest. On the other hand, Figures 6,7 and 8 display the evolution of the LOLE adequacy index, the evolution of the average yearly electricity price and of the capacity factors of the three candidate technologies.

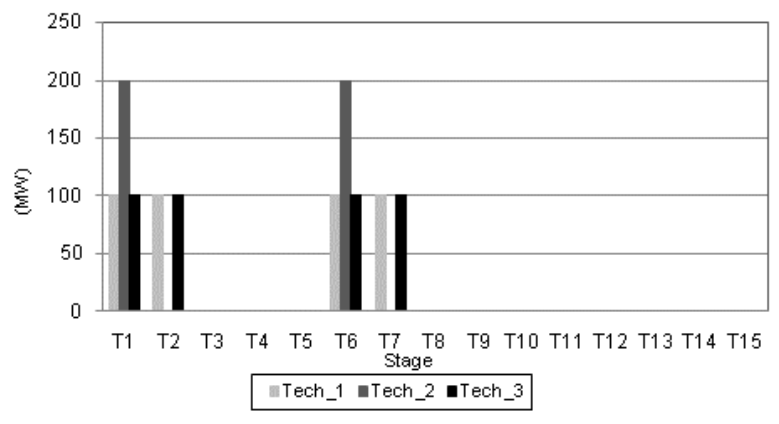

Fig. 4. Reference Case - Expansion plan obtained for GENCO 1.

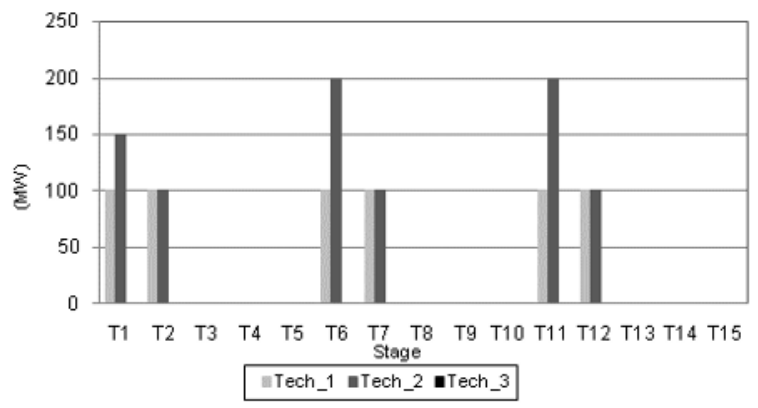

Fig. 5. Reference Case - Expansion plan obtained for GENCO 2. 


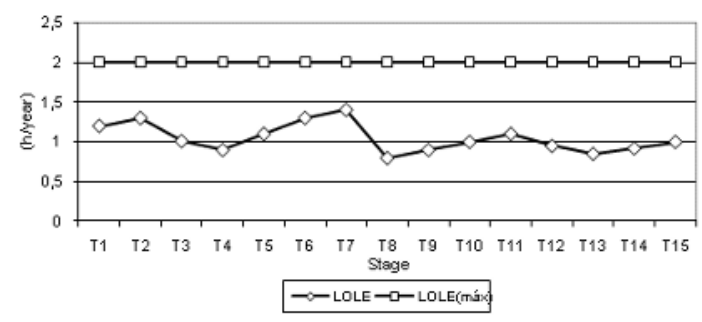

Fig. 6. Reference Case - Evolution of the LOLE adequacy index.

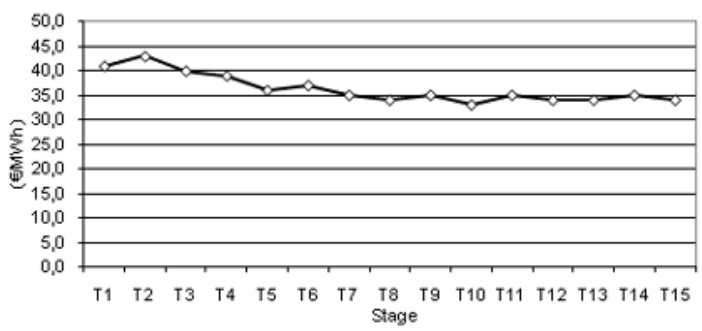

Fig. 7. Reference Case - Evolution of the yearly average electricity price.

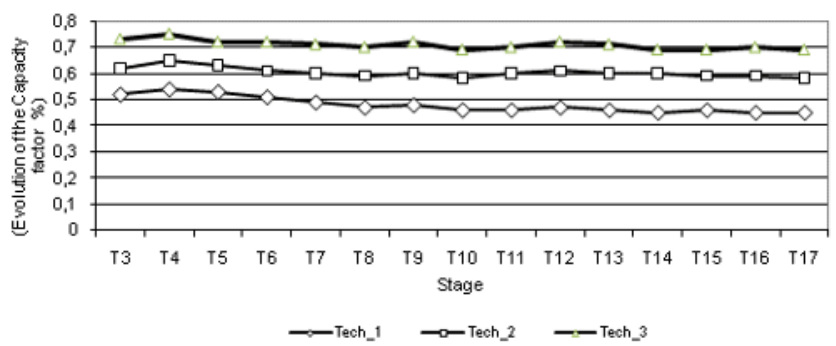

Fig. 8. Reference Case - Evolution of the capacity factors of the three candidate technologies.

Given the mentioned concentration of investment decisions on years 1, 2, 6, 7, 11 and 12, these new stations are commissioned two years afterwards, that is, at years $3,4,7,8,12$ and 13, given the construction period of 2 years that was specified. This explains the behavior of the LOLE. For instance, the value of LOLE increases from year 1 to 2 , and then decreases till year 4 given the extra generation capacity. As the demand increases, the LOLE tends to increase again till year 7 and then decreases again. Regarding the electricity price, Figure 7 shows that it displays a reduction along the planning horizon. This is explained by the more reduced operation costs of the new additions regarding the initially existing generation mix, together with the commissioning of new hydro stations and wind parks, as detailed in Section 5.1. In this case, the average electricity price is 36,3 $€ \mathrm{MWh}$ while the reference price in the starting period of the simulation was $41,0 € / \mathrm{MWh}$.

As mentioned above, these results were used as the reference ones so that we can make a number of comparisons with the output of the studies described in the next Sections. These studies can be understood as a sensitivity analysis in order to evaluate the impact on the evolution of the system, namely on the electricity price, of increasing the installed capacity in new wind parks by $50 \%$ regarding the values used above, of doubling the installed capacity in new wind parks and finally of 
doubling not only the installed capacity in new wind parks but also in new hydro stations. Given that the focus is on the impact on the electricity price, in the next sections we will present the results of the evolution of the yearly average electricity price and of the capacity factors of the three candidate technologies.

\subsection{Results - Case 1 - Increasing the installed capacity in new wind parks by $\mathbf{5 0 \%}$}

In this simulation, we increased the installed capacity in wind parks along the planning horizon. This capacity was $600 \mathrm{MW}$ in the reference case, described in Section 5.2 and it was now increased to $900 \mathrm{MW}$, with the following commissioning plan: $150 \mathrm{MW}$ in year 2, $150 \mathrm{MW}$ in year 4, 225MW in year 7, $150 \mathrm{MW}$ in year 10 and finally, 225 MW in year 12. Regarding the values used in the reference case, this means we maintained the commissioning years and increased the installed capacities by $50 \%$.

Regarding the results that were obtained with the long term simulation, Figure 9 shows the evolution of the average electricity prices along the horizon and Figure 10 displays the capacity factors of the three candidate technologies. On the other hand, the expansion plans obtained by the three generation agents were not altered regarding the ones in the reference case but, due to the increase of wind capacity by $300 \mathrm{MW}$, the average electricity price displays a small reduction from $36,3 € / \mathrm{MWh}$ in the reference case to $35,7 € / \mathrm{MWh}$, corresponding to $1,7 \%$. The capacity factors of the three candidate technologies displayed in Figure 10 also show a small reduction when compared with the values in Figure 8. For Tech_1 the capacity factor reduced by 2,7 \%, for Tech_2 it decreased by $2,5 \%$ and for Tech_3 the capacity factor reduced by $2,9 \%$ regarding the values obtained to the reference case.

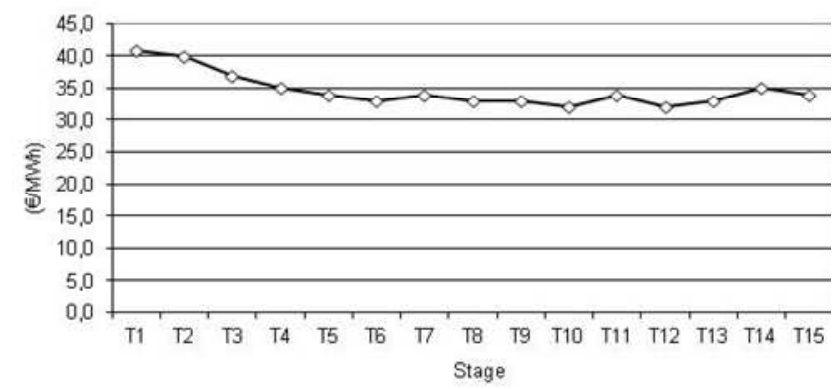

Fig. 9. Case 1 - Evolution of the yearly average electricity price.

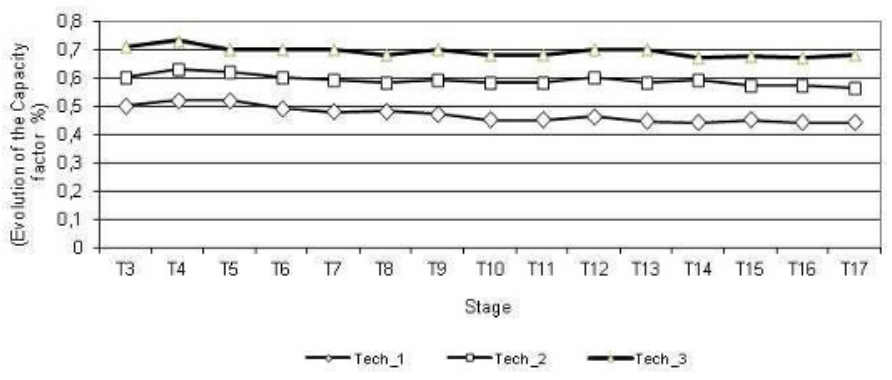

Fig. 10. Case 1 - Evolution of the capacity factors of the three candidate technologies. 


\subsection{Results - Case 2 - Doubling the installed capacity in new wind parks}

In this exercise we admitted that the installed capacity in wind parks to be commissioned along the planning horizon was doubled regarding the value of $600 \mathrm{MW}$ used in the reference case, that is it was increased to $1200 \mathrm{MW}$ along the horizon. On the other hand, we used the same commissioning years as in the simulation reported in Section 5.2. The evolution of the electricity price is shown in Figure 11 and its average value gets reduced by $2,8 \%$ regarding the reference case. On the other hand, the expansion plans of the three generation agents include now more reduced capacities to install along the horizon. For GENCO_1 there is a reduction of $100 \mathrm{MW}$, for GENCO_2 there is a reduction of $150 \mathrm{MW}$ and for GENCO_3 the new installed capacity gets reduced by $100 \mathrm{MW}$.

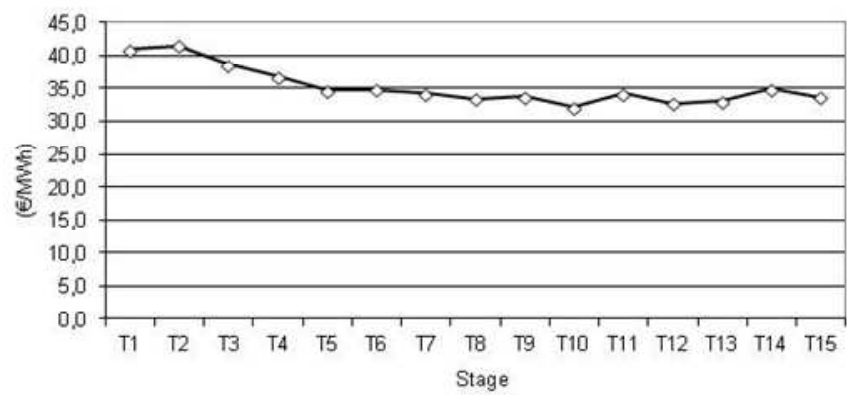

Fig. 11. Case 2 - Evolution of the yearly average electricity price.

Given the increased wind capacity, the capacity factors of the three candidate technologies also get reduced. Figure 12 displays the evolution of these capacity factors and from these values it is possible to conclude there is a reduction of 4,9\% for Tech_1, of 4,6 \% for Tech_2 and of 5,2\% for Tech_3 when compared with the values obtained in the reference case.

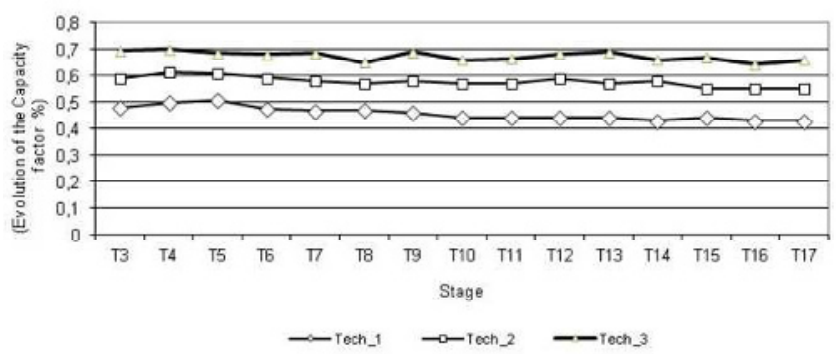

Fig. 12. Case 2 - Evolution of the capacity factors of the three candidate technologies.

\subsection{Results - Case 3 - Doubling the installed capacity in new hydro stations and in new wind parks}

Finally, we admitted that both the new installed capacity in wind parks and in hydro stations were doubled regarding the values used in the reference case. This means that for wind parks we admitted that $1200 \mathrm{MW}$ of new installed capacity will be added along the horizon instead of the $600 \mathrm{MW}$ admitted for the reference case and for hydro we considered $400 \mathrm{MW}$ of new reservoirs in year 4 and $200 \mathrm{MW}$ of run of river stations in the $8^{\text {th }}$ year. In all these situations we admitted that the schedule of the new additions was the same as indicated in Section 5.1.

The expansion plans that were now obtained for the three generation companies show there is a reduction of the new installed capacity of Tech_1, 2 and 3 regarding the reference case (from 1200 
MW to 1000 MW for GENCO_1, from 1450 MW to 1200 MW for GENCO_2 and from 1250 MW to $1100 \mathrm{MW}$ for GENCO_3). This investment reduction is obviously due to the larger injections from wind parks and from hydro stations that also contribute to reduce the market price. In fact, comparing the graphs in Figure 7 for the reference case and in Figure 13 it is clear that the electricity market price gets reduced, in this case by average value of $4,4 \%$. As a result, the capacity factors of the three candidate technologies also get reduced as it becomes clear when comparing the graphs in Figure 8 for the reference case with the results displayed in Figure 14. In average, the capacity factors reduced by $10 \%$ in the three candidate technologies.

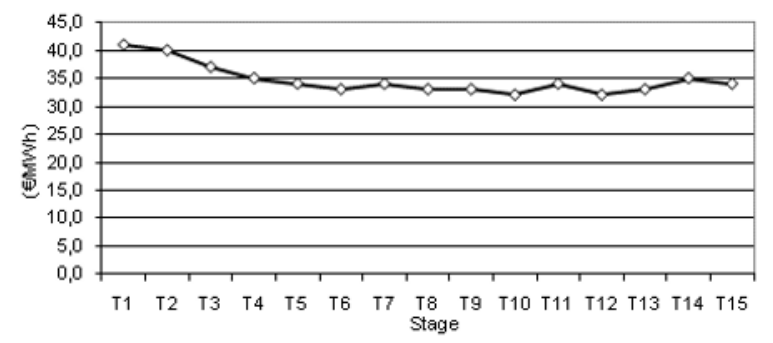

Fig. 13. Case 3 - Evolution of the yearly average electricity price.

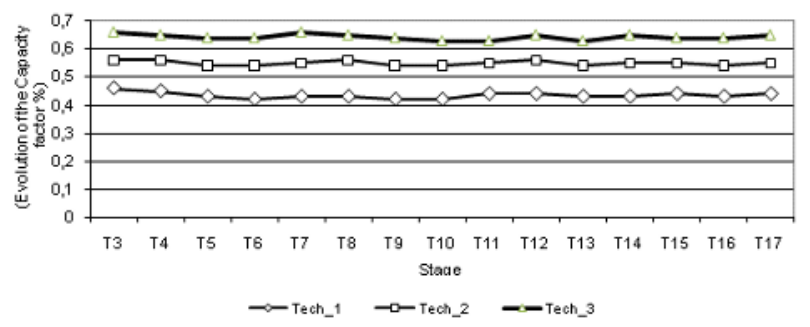

Fig. 14. Case 3 - Evolution of the capacity factors of the three candidate technologies.

\subsection{Comments}

The results obtained with these simulations, including the sensitivity analysis admitting the increased values of the installed capacity in wind parks and hydro stations, illustrate the impact of these increases on the electricity market prices. The prices obtained for the four analysis are displayed in Table 4 in terms of the yearly average values and of the global average value.

Given these reductions it gets clear that the profitability of existing stations, namely thermal stations can become compromised thus suggesting that regulatory agencies or other state boards should closely monitor this impact as new additions of infra marginal technologies to the power systems continue to increase. In fact, if adequate tariff and pricing approaches are not assumed it is possible that investments in traditional stations get reduced, thus compromising the security of supply namely in view of the larger presence of stations using volatile resources as wind and solar radiation. If such effect becomes relevant and puts the security of operation is risk, then it should be considered the creation of a capacity tariff to be remunerate traditional thermal technologies so that these investments can still be induced.

On the other hand, in several countries renewable stations are paid feed-in tariffs either fixed or eventually variable along the day to prize injections in off valley hours. If that is the case, the renewable source promoters would not feel any impact on their remuneration given the reduction of the market prices. In that case, the end consumers would pay an extra cost to subsidize renewable 
stations given the increased difference between the value of the feed-in tariffs and market price. This suggests that as a result of the increased wind power capacity the final price paid by end consumers would not decrease since it would be pressed by these extra subsidies.

Table 4. Yearly average electricity prices obtained for the four simulations.

\begin{tabular}{|c|c|c|c|c|}
\hline $\begin{array}{c}\text { Average } \\
\text { electricity price }\end{array}$ & $\begin{array}{c}\text { Base case } \\
(€ / \mathrm{MWh})\end{array}$ & $\begin{array}{c}\text { Case 1 } \\
(€ / \mathrm{MWh})\end{array}$ & $\begin{array}{c}\text { Case 2 } \\
(€ / \mathrm{MWh})\end{array}$ & $\begin{array}{c}\text { Case 2 } \\
(€ / \mathrm{MWh})\end{array}$ \\
\hline Year 1 & 41,0 & 41,0 & 41,0 & 41,0 \\
\hline Year 2 & 43,0 & 41,3 & 41,5 & 40,2 \\
\hline Year 3 & 40,0 & 39,6 & 38,6 & 37,1 \\
\hline Year 4 & 39,0 & 38,6 & 36,8 & 35,2 \\
\hline Year 5 & 36,0 & 35,6 & 34,7 & 34,0 \\
\hline Year 6 & 37,0 & 35,2 & 35,2 & 33,0 \\
\hline Year 7 & 35,0 & 34,0 & 34,2 & 34,0 \\
\hline Year 8 & 34,0 & 33,8 & 33,4 & 33,2 \\
\hline Year 9 & 35,0 & 34,4 & 33,8 & 33,1 \\
\hline Year 10 & 33,0 & 32,4 & 32,1 & 32,0 \\
\hline Year 11 & 35,0 & 34,2 & 34,2 & 33,9 \\
\hline Year 12 & 34,0 & 33,7 & 32,7 & 32,2 \\
\hline Year 13 & 34,0 & 33,6 & 33,1 & 33,1 \\
\hline Year 14 & 35,0 & 34,4 & 34,9 & 34,8 \\
\hline Year 15 & 34,0 & 33,8 & 33,6 & 33,7 \\
\hline Average & 36,3 & 35,7 & 35,3 & 34,7 \\
\hline
\end{tabular}

Given the increased amount of these subsidies some countries allow the renewable stations to bid in the market receiving the market price eventually plus a premium to prize their contribution to increase the liquidity of the market. This option already exists in Spain and it was recently under discussion in Portugal, with the argument that wind technology is already sufficiently mature to allow its direct participation in the market and also to reduce the amount of subsidies internalized in the tariffs paid by end consumers. It is clear that the increased presence of infra marginal technologies in the market contributes to reduce the market price that afterwards is paid to all dispatched stations. If that approach is introduced, this means that when bidding in the market wind power would be contributing to reduce the price and so to reduce its own profitability as well as compromising future investments. These effects together with the reduction of the attractiveness of investments in traditional thermal technologies suggests once again that these impacts should be adequately monitored on the long term so that regulatory or state agencies have enough insight on the problem namely to design adequate pricing schemes not to compromise the security of the system on the long term and the presence of renewables in the generation mix.

\section{Conclusions}

This paper described a long term simulation approach of the electricity market that is coupled with an investment scheduling problem to be solved by each generation agent. As a result, one can simulate on the long term the evolution of the electricity price, of the demand and of the capacity factors of the generation technologies in the mix associated with the identification of the most adequate investment plans of each generation agent.

This type of models can be used in a profitable way by several agents, namely by generation agents. Generation companies can use these long term simulations and the possibility of running sensitivity studies to build their expansion plans, while testing different possible behaviours of their competitors or considering different commissioning plans for hydro stations and wind parks. These simulations will give them more insight on the evolution of the power system so that more sounded and less risky investment decisions can be taken. 
It can also be used by regulatory or state agencies to study the impact on the system of the increased presence of renewable resources namely on the market price. This impact may influence the profitability of investments on traditional thermal stations eventually increasing the risk of the system not being able to accommodate the demand. It can also affect the profitability of investments in renewables themselves, namely if these stations (for instance, wind parks) start to bid in the market and be paid a tariff reflecting the market price. As a whole, these models and the corresponding results give more insight to the decision markers, for instance signalling that changes on pricing schemes should be introduced and designing such changes in a more adequate way.

\section{References}

1. A. Ford, "System dynamics and the electric power industry", System Dynamics Review, 13 (1997), pp. 57-85.

2. J. D. Sterman, Business Dynamics: Systems Thinking and Modeling for a Complex World, (McGraw-Hill, 2000).

3. A. Botterud, M. Korpås, K. Vogstad and I. Wangensten, "A Dynamic Simulation model for Long-term Analysis of the Power Market," in Proceedings of the 2002 Power Systems Computation Conference, PSCC, 25th -28th June 2002, Seville, Spain.

4. J. W. Forrester, "System Dynamics and the Lessons of 35 Years", in The Systemic Basics of Policy Making in the 1990s, Edt. K. B. De Greene, Sloan School of Management, MIT, USA, April 1991.

5. A. Botterud, Long-Term Planning in Restructured Power Systems, PhD Thesis, (Norwegian University of Science and Technology, Trondheim, Norway, November 2003).

6. T. Kadoya, T. Sasaki, S. Ihara, E. Larose, M. Sanford, A. K. Graham, C. A. Stephens, C. K. Eubanks, "Utilizing System Dynamics Modelling to Examine Impact of Deregulation on Generation Capacity Growth", in Proceedings of the IEEE, 93 (2005), pp. 2060 - 2069.

7. F. Olsina, F. Garces, H.-J. Haubrich, "Modelling Long-Term Dynamics of Electricity Markets", Energy Policy, 34 (2006), pp. 1411-1433.

8. A. J. C. Pereira, J. T. Saraiva, "A Decision Support Tool for Generation Expansion Planning in Competitive Markets using System Dynamics Models", in Proceedings of the 2009 IEEE PowerTech, Bucharest, Romania, June/July 2009.

9. A. J. C. Pereira, J. T. Saraiva, "Generation expansion planning (GEP) - A long-term approach using system dynamics and genetic algorithms (GAs)", Energy International Journal, 36 (2011), 5180-5199.

10. J.-B. Park, J.-H. Kim and K. Y. Lee, "Generation expansion planning in a competitive environment using a genetic algorithm", in Proceedings of the 2002 IEEE Power Engineering Society Summer Meeting, 3 (2002), pp. 1169 - 1172.

11. A. J. C. Pereira, J. T. Saraiva, "Generation Expansion Planning in Competitive Markets", in Proceedings of the 2007 IEEE PowerTech, Lausanne, Switzerland, July 2007.

12. Powersim Software AS, Powersim 2.5 User's Guide and Reference Manual, (Powersim Press, 1996).

13. Powersim Software AS, Powersim Studio 7 Academic, (Powersim Software AS, Bergen, Norway, 2006). 